SELECCIONES MATEMÁTICAS
Universidad Nacional de Trujillo
ISSN: $2411-1783$ (Online)
Vol. $02(01): 20-24(2015)$

Aproximación de Funciones Continuas con Rango Pre-compacto.

\title{
Approximation of continuous functions Pre- Compact Range.
}

Esptiben Rojas Bernilla*

Received, Feb. 15, 2015

Accepted, Jun. 15, 2015.

DOI: http://dx.doi.org/10.17268/sel.mat.2015.01.02

\begin{abstract}
Resumen
Es fundamental en teoría de aproximación, establecer condiciones para pasar de la convergencia puntual a la convergencia uniforme de funciones en ese sentido Hernández. [23] estableció algunos resultados relativos al problema de aproximar uniformemente funciones continuas con rango pre-compacto. En este trabajo daremos algunos nuevos resultados al respecto, estableciendo el concepto de $\mathcal{A}$ - separación debil a $F$, donde $\mathcal{A} \subseteq C(X)$ y $F \in C(X, E)$.
\end{abstract}

Palabras clave. Análisis, Topología, Aproximación.

\begin{abstract}
It is essential in theory approach, establish conditions to move from the convergence point of convergence uniform duties in this regard Hernández. [23] established some results relating to the problem of approximate uniformly continuous functions with pre-compact range. In this paper we will give some new results in this regard, establishing the concept of $\mathcal{A}$ - separation weak to $F$, where $\mathcal{A} \subseteq C(X)$ and $F \in C(X, E)$.

Keywords. Analysis, Topology, Approximation.
\end{abstract}

1. Introducción. En esta sección daremos algunas definiciones y terminología básicas que se usara en el resto del trabajo. En todo el trabajo se considerará $X$ un espacio de Hausdorff completamente regular no vació y $E$ un espacio de Banach real.

Denotaremos por,

$$
\begin{gathered}
C(X, E)=\{f: X \rightarrow F / f \text { es continua }\} \\
C^{*}(X, E)=\{f: X \rightarrow E / f \text { es continua y } X \text { es pre-compacto }\}
\end{gathered}
$$

Si $x \in E$ y $A \subseteq E$ definimos la distancia de $x$ al conjunto $A$ por

$$
d(x, A)=\inf \{\|x-a\|: a \in A\}
$$

donde $\|$.$\| denota la norma en E$ y además

$$
B_{\epsilon}(A)=\{x: x \in E, d(x, A)<\epsilon\} .
$$

Si $A$ es un subconjunto de un espacio topológico denotamos por $\bar{A}$ la clausura uniforme de $A$ en este espacio topológico.

\footnotetext{
*Facultad de Ciencias, Universidad de Magallanes, Avda. Bulnes 01855 Casilla 113 - D, Punta Arenas, Chile (esptiben.rojas@umag.cl).

This work is licensed under the Creative Commons Attribution-NoComercial-ShareAlike 4.0.
} 
Dotamos a $C(X, E)$ con la topología de la convergencia uniforme, así para cada $F \in C(X, E)$ y $\forall \epsilon>0$ un entorno básico para $F$ está definido por el conjunto de la forma

$$
N_{\epsilon}(F)=\{G: G \in \mathbb{C}(X, E),\|F(x)-G(x)\|<\epsilon \text { para todo } x \in X\}
$$

Si $F \in C(X, E), \mathcal{G} \subseteq C(X, E)$ y $S \subseteq X$ definimos

$$
d_{S}(F, \mathcal{G})=\inf \{\sup \{\|F(x)-G(x)\|: x \in S\}: G \in \mathcal{G}\}
$$

( nótese que $d(F, \mathcal{G})$ puede ser $+\infty$ )

Escribiremos $C(X)$ por $C(X, \mathbb{R})$.

Si $S \subset X$ y $F$ es una función sobre $X$ escribimos $\left.F\right|_{S}$ para la restricción de $F$ sobre $S$.

Si $\mathcal{G} \subseteq C(X, E)$ entonces $\mathcal{G} \mid S=\{G \mid S: G \in \mathcal{G}\}$.

Definición 1. Si $\mathcal{A} \subseteq C(X) \mathcal{G} \subseteq C(X, E)$, y a es un número cardinal definimos

$$
\langle\mathcal{G}, \mathcal{A}\rangle_{\alpha}=\left\{\sum_{i \in I} h_{i} G_{i} /|I|<\alpha, h_{i} \in \mathcal{A}, G_{i} \in \mathcal{G}\right\}
$$

Es claro que si $\mathcal{G}$ es un módulo sobre $\mathcal{A}$, con respecto a la multiplicación puntual de funciones entonces $\langle\mathcal{G}, \mathcal{A}\rangle_{\aleph} \subseteq \mathcal{G}$ y si $\mathcal{A}$ es un sub álgebra de $C(X)$ que contiene la unidad, entonces la inclusión se convierte en una igualdad.

Escribiremos $\langle\mathcal{G}, \mathcal{A}\rangle$ cuando no existe restricción sobre la cardinalidad del conjunto de índices.

Definición 2. Sea $\mathcal{A} \subset C(X)$ un subconjunto no vació $S$ de $X$ se llama un $\mathcal{A}$ - conjunto antisimétrico de $X$ si f es un elemento de $\mathcal{A}$ y la restricción de $f$ a $S$ es un valor real, entonces la restricción de $f$ a $S$ es constante. Así para álgebras reales $\mathcal{A}$ un conjunto antisimétrico, es igual a un conjunto donde todas las funciones del álgebra son constantes.

Se define la siguiente relación en $X$ si $p \in X$ y $q \in X$ diremos que $p \sim q$ cuando existe un conjunto $\mathcal{A}$ - antisimétrico $S$ que contenga a $p$ y $q$. Esta relación es de equivalencia en $X$, las clases de equivalencia son los conjuntos $\mathcal{A}$ - antisimétricos maximales de $X$. Luego define una única descomposición de $X$ en conjuntos disjuntos dos a dos, no vacíos.

Denotamos por $\mathcal{A}(X)$ la familia de todas los conjuntos $\mathcal{A}$ - conjunto antisimétrico de $X$

$$
\mathcal{A}(X)=\left\{S \subset X /\left.f\right|_{S}=\text { constante } \forall f \in \mathcal{A}\right\}
$$

Definición 3. Si $\mathcal{A}$ es un sub álgebra de $C(X, E)$ Decimos que $\mathcal{A}$ es Puntualmente Constante si $\mathcal{A}(X)=\{\{x\}: x \in X\}$

Definición 4. Una familia $\left\{h_{\alpha} / \alpha \in \mathbb{R}\right.$ de funciones continuas $h_{\alpha}: X \rightarrow I$ es llamada una Partición de la Unidad sobre $X$ si

1. Los soportes de $h_{\alpha}$ forma un cubrimiento cerrado finito de $X$

2. $\sum_{\alpha} h_{\alpha}(x)=1 \forall x \in X$

Sea $\left\{\mathbb{U}_{\beta} / \beta \in I\right\}$ es un cubrimiento abierto de $X$ se dice que la partición $\left\{h_{\alpha} / \alpha \in I\right\}$ de la unidad está subordinada a $\left\{\mathbb{U}_{\beta}\right\}$ si el soporte de cada $h_{\beta}$ esta contenido en algún $\mathbb{U}_{\beta}$.

Definición 5. Si $\mathcal{A} \subseteq C(X), \mathcal{G} \subseteq C(X, E)$ y $\alpha$ un número cardinal, diremos que $\mathcal{A} \alpha$-refina $\mathcal{G}$ cuándo para cada recubrimiento abierto $\mathbb{U}$ de $X$ de la forma

$$
\mathbb{U}=\left\{G_{i}^{-1}\left(B_{\epsilon}\left\{G_{i}\left(S_{i}\right)\right\}\right): G_{i} \in \mathcal{G}, S_{i} \in \mathcal{A}(X), i \in I\right\}
$$

existe una partición localmente finita de la unidad $\left\{h_{j}: j \in J\right\} \subseteq \mathcal{A}$ que está subordinado a $\mathbb{U}$, con $h_{i} \geq 0$ para todo $j \in J y|J|<\alpha$.

Diremos que $\mathcal{A}$ refina $\mathcal{G}$ cuando no existe restricción sobre la cardinalidad de J.

Definición 6. Sea $F \in C(X, E)$ y $\mathcal{A} \subseteq C(X)$, diremos que $\mathcal{A}$ separa $F$ cuándo para todo número $r$ y $t$ con $0<r<t$ y cada $S \in \mathcal{A}(X)$, existe una función $h \in \mathcal{A}$ y una constante real $p$ tal que $h\left(F^{-1}\left(\overline{B_{r}\{F(S)\}}\right) \geq p>0\right.$ y $h\left(X \backslash F^{-1}\left(B_{t}(\{F(S)\})\right)\right)=0$

Observación 1. Es importante notar que si $\mathcal{A}=C(X)$, entonces toda función $F \in C(X, E)$ está $\mathcal{A}-$ separado por $\mathcal{A}$.

Definición 7. Dado $F \in C^{*}(X, E)$, se dice que $\mathcal{A}$ - separa debilmente a $F$, cuando para toda $B(a, r) \subseteq$ E y $\delta>0$ existe $f \in \mathcal{A}$ tal que cumple:

1. $0 \leq f \leq 1$

2. $\left.f\right|_{F^{-1}(B(a, r))}=1$ y $\left.f\right|_{X-F^{-1}(B(a, r+\delta))}=0$ 
Sea $\mathcal{F} \subset C^{*}(X, E)$ se dice que $\mathcal{A}$ separa débilmente a $\mathcal{F}$ si $\mathcal{A}$-separa débilmente a $G$, para todo $G \in \mathcal{F}$

Definición 8. Sea $F \in C(X, E)$ y $\mathcal{A} \subseteq C(X)$, diremos que $\mathcal{A}$ separa $F$ cuándo para todo número $r$ y $t$ con $0<r<t$ y cada $S \in \mathcal{A}(X)$, existe una función $h \in \mathcal{A}$ y una constante real $p$ tal que $h\left(F^{-1}\left(\overline{B_{r}\{F(S)\}}\right) \geq p>0\right.$ y $h\left(X \backslash F^{-1}\left(B_{t}(\{F(S)\})\right)\right)=0$

Observación 2. Es importante notar que si $\mathcal{A}=C(X)$, entonces toda función $F \in C(X, E)$ está $\mathcal{A}-$ separado por $\mathcal{A}$.

Definición 9. Dado $F \in C^{*}(X, E)$, se dice que $\mathcal{A}$ - separa debilmente a $F$, cuando para toda $B(a, r) \subseteq$ E y $\delta>0$ existe $f \in \mathcal{A}$ tal que cumple:

1. $0 \leq f \leq 1$

2. $\left.f\right|_{F^{-1}(B(a, r))}=1$ y $\left.f\right|_{X-F^{-1}(B(a, r+\delta))}=0$

Sea $\mathcal{F} \subset C^{*}(X, E)$ se dice que $\mathcal{A}$ separa débilmente a $\mathcal{F}$ si $\mathcal{A}$-separa débilmente a $G$, para todo $G \in \mathcal{F}$

2. Algunos resultados previos. Denotaremos por $F-\mathcal{G}$ a la familia $\{F-G / G \in \mathcal{G}\}$

Lema 1. Si $\mathcal{A} \subseteq C(X), \mathcal{G} \subseteq C(X, E)$ y $F \in C(X, E)$.

1. Si $\mathcal{A} \alpha$-refina a $F-\mathcal{G}$, entonces $\left.d_{X}(F,\langle\mathcal{G}, \mathcal{A}\rangle)_{\alpha}\right) \leq \sup \left\{d_{S}(F, \mathcal{G}): S \in \mathcal{A}(X)\right.$. $\}$

2. Supongamos que $\mathcal{A}(X)$ es Puntualmente Constante y $\mathcal{G}$ contiene todas las funciones constantes. Si $\mathcal{A} \alpha$-refina $\{F\}$ entonces $d_{X}\left(F,\langle\mathcal{G}, \mathcal{A}\rangle_{\alpha}\right)=0$

Para la prueba ver [23].

Lema 2. Si $\mathcal{A} \subseteq C^{*}(X)$ y $\mathcal{G} \subseteq C^{*}(X, E)$. Entonces $d_{X}(F,\langle\mathcal{G}, \mathcal{A}\rangle)_{\aleph}=d_{X}\left(F,\langle\mathcal{G}, \overline{\mathcal{A}}\rangle_{\aleph}\right)$. Para la prueba ver [23].

Lema 3. Sea $\mathcal{A}$ es un subálgebra cerrada uniforme de $C^{*}(X)$ que contiene a la unidad y $F \in$ $C^{*}(X, E)$. Si $\mathcal{A}$ separa $F$ entonces $\mathcal{A} \aleph$-refina $\{F\}$. Para la prueba ver [23].

Usando los Lemas anteriores, se obtiene el siguiente resultado

Teorema 1. Si $\mathcal{A} \subseteq C^{*}(X), \mathcal{G} \subseteq C^{*}(X, E)$ es tal que para cada elemento de $\mathcal{A}(X)$ es Puntualmente Constante y $\mathcal{G}$ contiene las funciones constantes. Si $\mathcal{A}$ separa $F \in C^{*}(X, E)$, entonces $d_{X}(F,\langle\mathcal{G}, \mathcal{A}\rangle)=0$

Para la prueba ver [23].

3. Algunos nuevos resultados. Teorema 2. Si $\mathcal{A}$ separa a $F \in C^{*}(X, E)$. Además si $\mathcal{G} \subset C(X, E)$ un subálgebra que contiene a las constantes, entonces $\langle\mathcal{A}, \mathcal{G}\rangle_{0}$, aproxima uniformemente a $F$. Prueba:

Sea $\epsilon>0$ y como $F$ tiene rango pre-compacto, existe

$$
\left\{x_{1}, x_{2}, \ldots, x_{n}\right\} \in X \text { tal que } F(X) \subseteq B\left(F\left(x_{1}\right), \epsilon\right) \cup, \ldots, B\left(F\left(x_{n}\right), \epsilon\right)
$$

Denotando por $B_{j}=B\left(F\left(x_{j}\right), \epsilon\right)$ y $B^{j}=B\left(F\left(x_{j}\right), 2 \epsilon\right)$ y como $\mathcal{A}$-separa a $F \in C(X, E)$, se tiene que

$\forall j 1 \leq j \leq n$, existe $h_{j} \in \mathcal{A}$ tal que

- $0 \leq h_{j} \leq 1$

- $\left.h_{j}\right|_{F^{-1}\left(B_{j}\right)}=1$ y $\left.h_{j}\right|_{X-F^{-1}\left(B^{j}\right)}=0$

Luego $\forall x \in X$, se cumple que $\sum_{j=0}^{n} h_{j}(x)>0$. puesto que $x \in F^{-1}\left(B_{j}\right)$ para algún $j$.

Definiendo por recurrencia

$$
\begin{gathered}
g_{1}=h_{1} \\
g_{i}=\left(1-h_{1}\right)\left(1-h_{2}\right) \ldots\left(1-h_{i-1}\right) h_{i} \text { donde } 1<i \leq n
\end{gathered}
$$

Por inducción sobre $n \in N$ se demuestra que

$$
\sum_{i=1}^{n} g_{i}(x)=1, \quad \forall x \in X
$$

Tomando

$$
G(x)=\sum_{j=1}^{n} g_{j}(x) \widetilde{F\left(x_{i}\right)} \in\langle\mathcal{A}, \mathcal{G}\rangle_{N}
$$

donde $\widetilde{F\left(x_{i}\right)}$ es una constante.

Luego se tiene que :

$$
\|F(x)-G(x)\|=\left\|\sum_{j=1}^{n} g_{j}(x) F(x)-\sum_{j=1}^{n} g_{j}(x) \widetilde{F\left(x_{j}\right)}\right\| \leq \sum_{j=1}^{n} g_{j}(x)\left\|F(x)-\widetilde{F\left(x_{j}\right)}\right\|
$$


observando que $x \in F^{-1}\left(B_{j}\right)$ entonces $\left\|F(x)-\widetilde{F\left(x_{j}\right)}\right\|<\epsilon$, por lo tanto

Dado $F \in C^{*}(X, E)$ existe $G \in\langle\mathcal{A}, \mathcal{G}\rangle$ tal que $\|F-G\| \leq \epsilon$ para todo $\epsilon>0$. con lo que el teorema queda probado.

Teorema 3. Si $F \in C *(X, E), \mathcal{G} \subseteq C^{*}(X, E)$ y $\mathcal{A}$ separa débilmente a $F-\mathcal{G}$ entonces

$$
d_{\mu}\left(F,\langle\mathcal{G}, \mathcal{A}\rangle_{0}\right) \leq \sup \left\{d_{S}(F, \mathcal{G}): S \in \mathcal{A}(X)\right\}
$$

\section{Prueba:}

Sea $r=\sup \left\{d_{S}(F, \mathcal{G}): S \in \mathcal{A}(X)\right\}$

Si $r=+\infty$ no hay nada que probar.

Si $r<+\infty$ entonces, tomado $\epsilon>0, \forall S \in \mathcal{A}(X)$ existe $G_{S} \in \mathcal{G}$ tal que

$$
r \leq\left\|F(x)-G_{S}(x)\right\|_{S}<r+\epsilon
$$

donde $x_{j} \in S_{j}$. Como $A$ separa a $F-\mathcal{G}$ entonces $\forall j, 1 \leq j \leq n$ existe $h_{j}$ tal que

1. $0 \leq h_{j} \leq 1$

2. $\left.h_{j}\right|_{\left(F-G_{S_{j}}\right)^{-1}\left(B\left(F-G_{S_{j}}\right)\left(x_{j}\right), \epsilon\right)}=\left.1 \quad \mathrm{y} \quad h_{j}\right|_{X-\left(F-G_{S_{j}}\right)^{-1}\left(B\left(F-G_{S_{j}}\right)\left(x_{j}\right), 2 \epsilon\right)}=0$

Luego $\sum_{1 \leq j \leq n} h_{j}>0$ puesto que $x \in\left(F-G_{S_{j}}\right)^{-1\left(B\left(F-G_{S_{j}}\right)\left(x_{j}\right), \epsilon\right)}$.

Definiendo por recurrencia

$$
\begin{gathered}
g_{1}=h_{1} \\
g_{i}=\left(1-h_{1}\right)\left(1-h_{2}\right) \ldots\left(1-h_{i-1}\right) h_{i} \text { donde } 1<i \leq n
\end{gathered}
$$

Por inducción sobre $n \in N$ se demuestra que

$$
\sum_{i=1}^{n} g_{i}(x)=1, \quad \forall x \in X
$$

Tomando $G=\sum_{1 \leq j \leq n} h_{j} G_{S_{j}}$ se demuestra como el teorema anterior, que $\|F-G\| \leq \epsilon$, con lo que termina la prueba.

Corolario 1. Sea $F \in C *(X, E)$ de manera que $\mathcal{A}$ separa a $\{F-G: G \in \mathcal{G}\}$. Si $\mathcal{A}$ es puntualmente constante y $\mathcal{G}$ aproxima puntualmente a $F$, entonces $\langle\mathcal{G}, \mathcal{A}\rangle_{0}$ aproxima uniformemente a $F$.

\section{Prueba:}

Por el teorema anterior

$$
d_{\mu}\left(F,\langle\mathcal{G}, \mathcal{A}\rangle_{0}\right) \leq \sup \left\{d_{S}(F, \mathcal{G}): S \in \mathcal{A}(X)\right\}
$$

Como $S=\{x\}$ y si $\mathcal{G}$ aproxima puntualmente a $F$, luego $d_{\{x\}}(F, \mathcal{G})<\epsilon$ para todo $\epsilon>0, x \in S$ Por lo tanto $d_{\mu}(F,\langle\mathcal{G}, \mathcal{A}\rangle)<\epsilon$.

Lo que significa que $\langle\mathcal{G}, \mathcal{A}\rangle_{0}$ aproxima uniformemente a $F$.

Referencias

[1] F. Anderson. Approximation in systems of real-valued continuous functions.Trans.Amer.Math.Soc. 103 (1962) $249-271$

[2] Bartle, Robert G.: The Elements of Real Análysis, Segunda Edición, New York,Editorial Wiley,(1976),90-192,315-346.

[3] E. Bishop: A generalization of the Stone-Weierstrass Theorem,Pacific J.Math.J.11 (1961).777-783.

[4] R.L.Blair Extensions of Lebesgue sets and real - valued functions, Czechoslovak Math.J. 31 (1981). 63-74.

[5] J.Blasco, L.Molto. On the uniform clasure of a linear space of bounded real-valued funtionsAnnali di Matematica Pura ed Applicata 134 (4)1983, 233-239.

[6] J.Blasco. Hausdorff compactification and Lebesgue set. Topology and its Application. 15 (1983) 111-117.

[7] J.Blasco. Conjuntos de Lebesgue y compactaciones de un espacio topologico.Rev. Real Acad. de Ciencias Exactas, Fisicas y Naturales de Madrid. 78 (1984) 295-200.

[8] J.Blasco. Complete bases and Wallman realcompactification.Proc.Amer.Soc. 75 (1979),114-117.

[9] B.Brosowski; F.Deutsch. An Elementary Proof of the Stone-Weierstrass Theorem.Proceedings of the American Mathematical Society, Vol 81, Na1 (1981),89-92.

[10] R.B.Burkel: Bishop's Stone-Weierstrass Theorem, Amer.Math.Monthly 91 (1984). 22-32.

[11] T.Carleman: Sur un Théoreme de Weierstrass.1927

[12] Jean-Etienne Rombaldi. Sur les theoremes de Stone-Weierstrass et de Korovkin- 9 junio 2003.

[13] Carothes,N.L.: A Short Course on Aproximation Theory, Department of Mathematics and Statistics, Bowling green State University,(1998).

[14] D.A.Edwards: A Short proof of a Theorem of Machado, Math.Proc. Cambridge Philos.Soc.99 (1986), $111-114$.

[15] R. Engelking: General Topology, Polish Scientific, Warszawa 1977. 
[16] M.Estrada: Los Teoremas de Ascoli y Stone-Weiertrass, su aplicación en el Análisis Funcional.(1998) Tesis de Licenciatura.UNPRG.

[17] J.Galindo, M. Sanchis. Stone-Weierstrass Type Theorems For Group-Valued Fuctions.

[18] J. Gomez. 'Algebra de Funciones Continuas Intermedias entre $C^{*}(X)$ y $C(X)$ Tesis Doctoral. Universidad de Valladolid.1997.

[19] L.Gillman and M.Jerison: Rings of continuous functions, Van Nostrand, Princenton.1960.

[20] A.Hager. On inverse-closed subalgebras de C (X). Proc. London. Math. Soc.III Ser.19 (1969) 233-257

[21] A. Hager. An approximation tecnique for real-valued functions, General Topoly and its Applications, 1 1971, $127-133$.

[22] M.Henriksen. Unsolved problems on algebraic aspects de $C(X)$.Lecture Notes in Pure and Appl.Math, 95,Dekker, New York, 1985.

[23] S. Hernández. Approximation and extension de continuos fuctions

[24] S. Hernández. Algebra de Funciones Continuas Tesis Doctoral . Universidad de Valencia. 1983.

[25] E. Hewitt: Certain representation of the Weierstrass approximation theorem, Ducke Math., 14 (1947), $419-427$.

[26] R.Jewett. A Variation on the Stone-Weiertrass Theorem.Proceedings of the American Mathematical Society,Vol 14, N55 (1963),690-693.

[27] S.Kakutani. Concrete representation of abstract (M)-space,Annals of Math., 42 (1941), 994-1024.

[28] S.Machado: On Bishop's generalization of the Stone-Weierstrass Theorem, Indag.Math.39 (1977).218-224.

[29] S.Mrówka. On some approximation Theorems, Nieuw Archief voor Wiskunde,XVI (1968), 94-111.

[30] F.Montalvo: Uniform approximation theorems for real-valued continuous function, Topology and its Application 45 (1992)145155 North-Holland.

[31] F.Montalvo y M.I.Garrido. On Some Generalizations Of The Kakutani-Stone And Stone-Weierstrass Theorems,Acta Math.Hung.(1993), 199-208.

[32] F. Montalvo Y M.I. Garrido: Generation of Uniformly Closed Algebras of FunctionsPositivity, $82005,8195$.

[33] F.Montalvo Y M.I.Garrido. Algebraic Properties of the Uniform Closure of Space of Continuous Function Annals of the New York . Academy of Sciences. Volumen 788,(1996)

[34] F.Montalvo y M.I. Garrido. Uniform approximation theorems for real-valued continuous functions. Topology and its Application 45 (1992)145-155.North-Holland.

[35] F.Montalvo y M.I. Garrido. Generation of the uniformly continuous functions.Topology and its Application, $1372004,167174$.

[36] M.I.Garrido: Aproximación Uniforme en Espacios de Funciones Continuas, Departamento de Matemáticas -Universidad de Extremadura (1990)

[37] A.Pinkus. Weierstrass and Approximation Theory

[38] A.Pinkus. Density in Approximation Theory-4 julio-2004.

[39] A.Pinkus. Density Methods And Results in Approximation Theory.Orlicz Centenary Volume. Banach Center Publication, Volume 64.(2004)

[40] J.Prolla: Weirstrass-Stone the theorem, Velag Peter Lang GmbH ,Frankfurt am Main 1993

[41] T.J. Ransford. One Short elementary proof of the Stone-Weierstrass - Bishop Theorem, Math, Proc. Cambridge Philos.Soc. 96(1984), 309-311.

[42] E.Rojas. Aproximación en Espacios de Funciones Continuas, Trabajo de Investigación -DEA, Universidad Jaume I,Castellón (2006).

[43] W. Rudin. An'alisis Funcional Editorial Reverté, S.A.-1979.

[44] Y. Sterfeld. Dense Subgroups of $C(K)$ Stone-Weierstrass Type Theorems for GroupsConstructive Approximation. 6:339-351 (1990).Springer- Verlag New York Inc.

[45] Y. Sternfeld, Y. Weit. An Approximation theorem for vector-valued function. In: Geometric Aspects Functional AnalysisLecture Notes in Mathematics. Belin: Springer-Verlag - 1998.

[46] M.H.Stone, A generalized Weierstrass approximation theorem Math. Magazine,21 (1948),167-184, $237-254$.

[47] K.Weierstrass. Sur la possibilité dúne représentation analytique des fonctions dites arbitraires dúne variable réelle.J.Math.Pure et Appl. 2 (1886) 115-138. 